\title{
Core Overshoot and Nonrigid Internal Rotation of Massive Stars: Current Status from Asteroseismology $\dagger$
}

\author{
Conny Aerts ${ }^{1,2}$ \\ ${ }^{1}$ Instituut voor Sterrenkunde, Katholieke Universiteit Leuven, Celestijnenlaan 200D, \\ B-3001 Leuven, Belgium \\ ${ }^{2}$ IMAPP, Radboud Universiteit Nijmegen, P.O. Box 9010, 6500 GL Nijmegen, the Netherlands \\ email: conny@ster.kuleuven. be
}

\begin{abstract}
Seismic estimates of core overshoot have been derived from extensive high-precision photometric and spectroscopic ground-based (multisite) campaigns for five main-sequence Btype stars. For three of these, the ratio of the near-core rotation frequency to the surface rotation frequency could be estimated as well, from the identified oscillation modes. We summarise these seismic results obtained for B stars. Now that the technique of asteroseismology was proven to work for probing the interior of massive stars, we expect a drastic increase in the precision of the structure parameters from the space missions CoRoT and Kepler, as well as from currently ongoing ground-based campaigns, in the coming years.
\end{abstract}

Keywords. stars: early-type - stars: oscillations - stars: evolution - stars: interiors - convection - line: profiles - techniques: spectroscopic - techniques: photometric - methods: data analysis stars: fundamental parameters

\section{The goals of asteroseismology of massive stars}

Asteroseismology is a modern branch within stellar astrophysics whose goal is to improve stellar evolution models by exploiting the character of stellar oscillations. For an extensive recent review, with specific emphasis on the synergy between asteroseismology and interferometry, we refer to Cunha et al. (2007). With respect to massive stars, the largest unknowns in their interior structure are the amounts of rotational mixing and of core convective overshooting. These two phenomena are crucial for the evolution of $\mathrm{O}$ and B stars, while they are poorly understood and hardly calibrated from observational data. Parameterised formulae are used to describe these two physical processes, but most of the current observational data are not accurate enough to provide a stringent test of them. The goal is to improve this situation from asteroseismology by considering, besides classical high-precision spectroscopy and interferometry, extensive time series of (multicolour) photometry and high-resolution spectroscopy to derive the details of the stellar oscillation properties. The latter are indeed an optimal probe of the interior physics of stars. Immense progress has been made in this field the last decade, see e.g. the summary by Kurtz (2006).

For a spherically symmetric star, each oscillation mode is characterised by its cyclic frequency $\nu_{n, \ell, m}$ and wavenumbers $(n, \ell, m)$, where $n$ is the radial order of the mode and $(\ell, m)$ are the wavenumbers of the spherical harmonic describing the angular part of the eigenfunction (see, e.g., Cunha et al. 2007). The observational part of an asteroseismic

$\dagger$ A large part of this research was performed within the Belgian Asteroseismology Group (BAG): http://www . asteroseismology.be, in particular within an intensive Leuven-Liège collaboration. 
study comes down to the derivation of $\nu_{n, \ell, m}$ and of the identification of $(\ell, m)$ from time series analysis. The theoretical part consists in the prediction of these quantities from a dense grid of stellar structure models.

Asteroseismology has the potential to derive high-precision (1-5\%) estimates of the fundamental parameters of massive stars by fitting observed identified oscillation frequencies and to derive the core overshoot parameter as well as to estimate the nonrigidity of the internal rotation. We show here that this has been achieved for a few carefully selected slowly-rotating B stars in the past few years.

\section{Procedure for core overshoot estimation}

Several independent evolution and oscillation codes have been developed. In preparation of the CoRoT space mission, seven evolution codes have been compared in great detail. Only very minor differences due to the different numerical tools were found, provided that the same assumptions on the input physics of the models (equation of state, opacities, convection, etc.) and their physical parameters were made (Monteiro et al. 2006). This led to very similar seismic predictions for the stellar models and implies negligible theoretical uncertainty in the seismic interpretation of measured oscillation frequencies compared to the uncertainty induced by the present-day data, including those expected from CoRoT (Moya et al. 2008). Differences in the theoretical interpretation of measured oscillations thus only come from different adopted input physics of the models.

For $\mathrm{O}$ and $\mathrm{B}$ stars, standard stellar models typically cover a 5D parameter space, with input parameters the stellar mass $M$, the stellar age, the hydrogen fraction $X$, the metallicity $Z$ and the core overshoot parameter $\alpha_{\mathrm{ov}}$ which expresses the thickness of the overshoot region around the stellar core. The overshoot parameter is usually expressed in terms of the local pressure scale height $H_{\mathrm{p}}$. Different descriptions of the temperature gradient and of the efficiency of the mixing in the overshoot region are in use and, thus, there is a theoretical uncertainty in the derived value of $\alpha_{\mathrm{ov}}$.

In order to estimate $\alpha_{\text {ov }}$ from fitting of measured oscillation frequencies, one usually adopts a forward modelling method. This consists first of all of the computation of a very dense 5D grid of stellar models covering the (uncertain!) position of the star in the HR diagram, or, preferably in the less uncertain $\left(T_{\text {eff }}, \log g\right)$ diagram. Secondly, the oscillation frequencies $\nu_{n, \ell, 0}$ and mode excitation for the grid models are computed. Subsequently, the quantum numbers $(\ell, m)$ of the detected frequencies must be derived from the data (e.g. Briquet \& Aerts 2003, Zima 2006). Finally, a match between the observed frequencies of the $m=0$ modes and those predicted by the models is made. For observed isolated frequencies with $m \neq 0$, this requires knowledge of the rotation frequency $\Omega(r)$ inside the star — see Eq.(3.1) discussed further on. As a good initial approximation, one assumes rigid internal rotation and derives $\bar{\Omega}$ from the measured $v \sin i$ and an estimate of the inclination angle from the mode identification (Mazumdar et al. 2006). If the inclination angle or the average rotation frequency cannot be estimated, then the $m \neq 0$ modes cannot be used in the seismic modelling.

A successful match is one for which the differences in observed and theoretical $m=0$ frequencies is less than the accuracy of the measured identified mode frequencies. In that case, the models are sufficiently sophisticated in terms of their input physics to approximate the star, and one obtains a very precise seismic estimate of all the fundamental stellar parameters, including mass and age. If no satisfactory match is obtained, the conclusion must be that the input physics of the models is too different from reality. In the latter case, one can use the oscillation information to try and improve this input physics. 
The situation may also occur that a good frequency match is achieved, but that (some of) the measured frequencies are predicted to be stable. That also allows to improve the input physics. Such a situation was encountered for the star HD 29248 whose detected oscillations pointed out a too low OPAL opacity value in the driving region (Pamyathnykh et al. 2004) or in the entire star (Ausseloos et al. 2004). This problem, as well as the occurrence of B-type pulsators in the Magellanic Clouds, have been solved meanwhile by considering recent OP opacity tables in combination with the new solar mixture (Miglio et al. 2007). Changes in the opacities typically have a large effect on the mode excitation as the driving is due to the $\kappa$ mechanism, but not on the frequency values as these are mainly determined by the density structure within the star.

\section{Nonrigidity of the internal rotation}

The rotation of a star implies that the oscillation frequencies are split into multiplets of the same $\ell$ but with different $m$, compared to the non-rotating case where all modes of the same $\ell$ and different $m$ have the same frequency value (e.g. Cunha et al. 2007). Thus, once the fundamental stellar parameters are fixed from a frequency match of the $m=0$ modes, one is able to exploit such frequency splittings, if multiplets or parts thereof are detected. The frequency spectra of the three B stars where this is the case are shown in Fig. 1. It can be derived from this figure that the radial mode, which is the radial fundamental for all three stars, occurs at lowest, middle and highest frequency for HD 29248, HD 129929 and HD 157056, respectively. Since the three stars have similar mass, this thus provides the order of age of these objects, which is compatible with their luminosity class. The multiplet structures seen in HD 129929 and HD 29248 are such that rotational splitting does not mix up frequencies of different radial order. HD 157056 (equatorial rotation velocity of about $30 \mathrm{~km} \mathrm{~s}^{-1}$, see Table 1), on the other hand, is a case where the rotation frequency is such that the multiplets are at the limit of being well separated. In particular, the $\ell=2, m=0$ mode is not observed for this star, but its frequency can be estimated from the three other frequencies of the $\ell=2$ multiplet, as they are identified as $m=-1,1,2$ (Briquet et al. 2007). Larger splitting would have implied difficulties to interpret the observed frequencies in terms of their $(\ell, m)$ values.

To test the rigidity of the rotation, so-called kernels $K_{n, \ell, m}(r)$ are computed (see, e.g., Cunha et al. 2007). These are functions over the radial distance inside the star which capture the relative sensitivity of the different mode frequencies to the internal properties of the star, such as the rotation, the kinetic energy density, etc. The frequency splittings are dependent on rotational kernels through

$$
\nu_{m}=\nu_{0}+m \int_{0}^{R} K_{n, \ell, m}(r) \frac{\Omega(r)}{2 \pi} \frac{\mathrm{d} r}{R},
$$

where $\nu_{0}$ is the frequency in the absence of rotation and where we assume $\Omega$ to depend only on the radial distance $r$ to the stellar centre. Thus, one can exploit the observed splittings within multiplets by comparing them with those predicted from Eq. (3.1). Usually, it is first tested if one can fulfil Eq.(3.1) with $\Omega$ constant throughout the star by using all the observed multiplets. If the data are in conflict with this assumption, the rotation cannot be rigid. Ideally, one would then want to invert the integral in Eq.(3.1) to derive $\Omega(r)$. This can only be done if many well-identified multiplets whose kernels have different probing power in the stellar interior are detected. This is the case for the Sun, but for no other main-sequence star so far. For the three B stars shown in Fig. 1, we only have splitting values from two different multiplets. As a result, we can only test how much $\Omega(r)$ near the core differs from the one in the envelope, e.g. by assuming a linear slope. This is how the values listed in Table 1 were derived. 

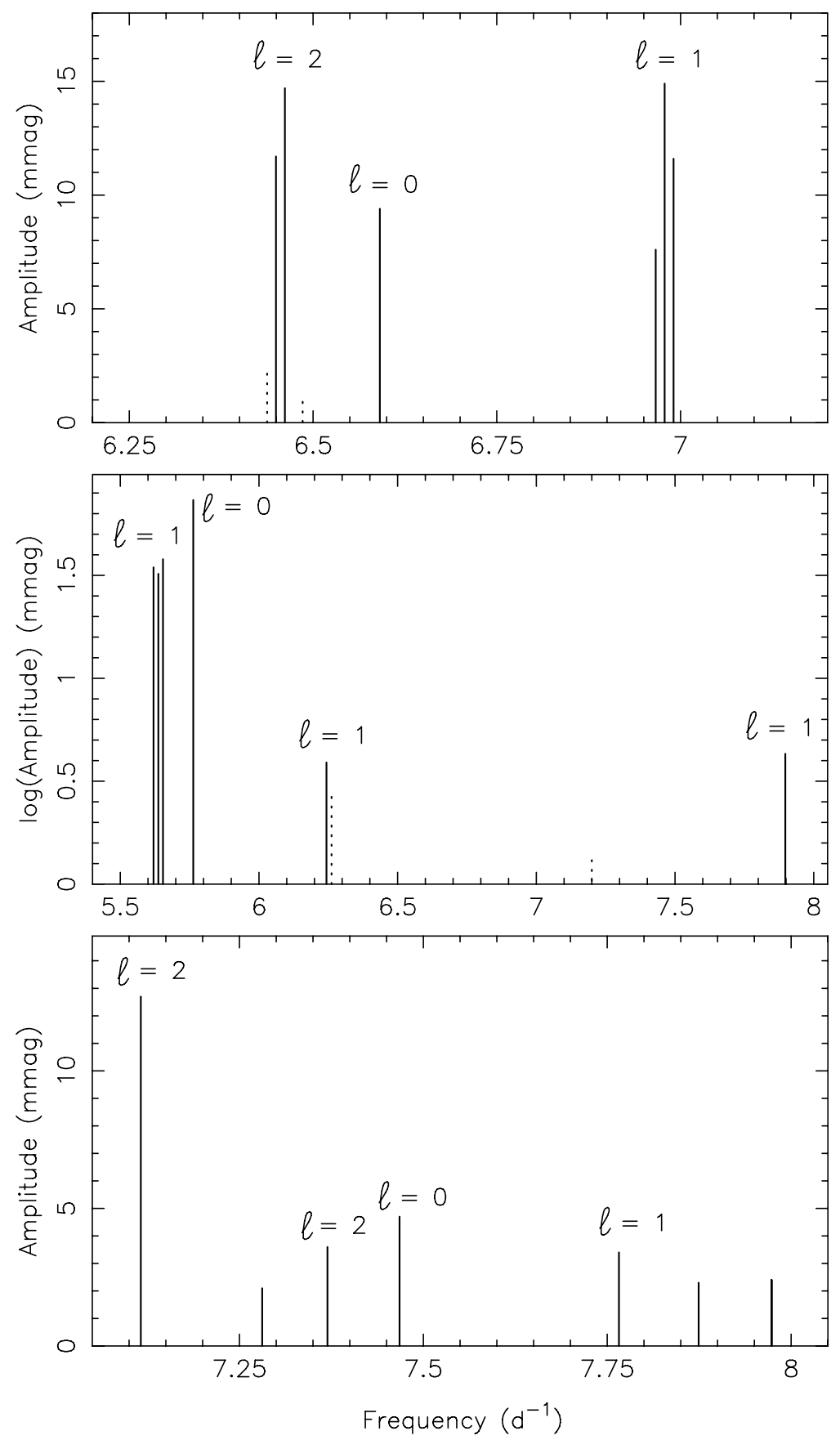

Figure 1. Schematic frequency spectra derived from photometric data of the $\beta$ Cep stars HD 129929 (top), HD 29248 (middle) and HD 157056 (bottom), reproduced from Aerts et al. (2004), De Ridder et al. (2004) and Handler et al. (2005), respectively. The lower and higher frequency ranges of HD 29248 do not contain identified modes and have been omitted for visibility reasons. The identified degrees are indicated. Full lines indicate frequencies above $4 \sigma$, while dotted lines represent frequencies present in the data but with significance below $4 \sigma$. Note the different axis scales. 
Table 1. Summary of the seismic results obtained so far. Reference numbers refer to (1): Aerts et al. (2006), (2): Pamyatnykh et al. (2004) and Ausseloos et al. (2004), (3): Mazumdar et al. (2006), (4): Aerts et al. (2003, 2004) and Dupret et al. (2004), (5): Briquet et al. (2007).

\begin{tabular}{llrlccc}
\hline Ref. & Star & Mass $\left(\mathrm{M}_{\odot}\right)$ & SpT & $\alpha_{\text {ov }}\left(\mathrm{H}_{\mathrm{p}}\right)$ & $\Omega R\left(\mathrm{~km} \mathrm{~s}^{-1}\right)$ & $\Omega_{\text {core }} / \Omega_{\text {env }}$ \\
\hline$(1)$ & HD 16582 & $10.2 \pm 0.2$ & B2 IV & $0.20 \pm 0.10$ & $28(14 ?)$ & \\
$(2)$ & HD 29248 & $9.2 \pm 0.6$ & B2 III & $0.10 \pm 0.05$ & $6 \pm 2$ & $\sim 5$ \\
$(3)$ & HD 44743 & $13.5 \pm 0.5$ & B1 III & $0.20 \pm 0.05$ & $31 \pm 5$ & \\
$(4)$ & HD 129929 & $9.4 \pm 0.1$ & B3 V & $0.10 \pm 0.05$ & $2 \pm 1$ & 3.6 \\
$(5)$ & HD 157056 & $8.2 \pm 0.3$ & B2 IV & $0.44 \pm 0.07$ & $29 \pm 7$ & $\sim 1$ \\
\hline
\end{tabular}

It is evident that the seismic tuning of the internal rotation is very dependent on the frequency accuracy, besides the nature of the probing power of the detected mode kernels. The frequency accuracy is mainly a function of the total time base of the data and also of the number of data points (i.e. the duty cycle). It is of order $10^{-6} \mathrm{~d}^{-1}$ for HD 129929 (21 years of low-duty single-site data), $10^{-5} \mathrm{~d}^{-1}$ for HD 29248 (150 days of high-duty 11-site data), and $10^{-4} \mathrm{~d}^{-1}$ for HD 157056 (5 months of moderate-duty 3-site data). Long-term monitoring is in any case required to probe the internal rotation of massive stars.

\section{Summary of the seismic results so far}

The procedures outlined in the previous two sections were successfully applied to five B-type pulsators so far. Many other, often extensive but failed attempts to derive $\alpha_{\text {ov }}$ are omitted here, in particular all those of rapidly rotating B and Be stars. Lack of secure mode identification is almost always the reason for the failure, besides frequency alias confusion. The results for the stars where $\alpha_{\text {ov }}$ could be tuned are summarised in Table 1 .

We point out that the quoted errors for the mass and core overshoot parameter do not include the theoretical uncertainties due to the changes of input physics of the models. The adopted opacities and metal mixtures have changed over the past few years, between the applications to HD 129929 and HD 157056, as a consequence of better opacity computations and a change in the solar mixture (Asplund et al. 2005). The effect of a change in the metal mixture was investigated for the case of HD 129929 by Thoul et al. (2004) and it was found that the new solar mixture generally leads to somewhat higher $\alpha_{\text {ov }}$ compared to the one obtained from the old solar mixture (Grevesse \& Noels 1993). The results of $\alpha_{\mathrm{ov}}$ for the first four stars in Table 1 were done with the old solar mixture and are therefore probably lower limits, but the modelling would have to be redone to make a quantitative assessment of this effect.

The results mentioned in Table 1 are of very high quality compared to what can be achieved from classical methods not using oscillations, even when considering the yet unquantified theoretical uncertainties due to the metal mixture. This becomes obvious when comparing the seismic results with those obtained from eclipsing binaries or from cluster isochrone fitting. Claret (2007) recently compiled results for eclipsing binaries and obtained $\alpha_{\text {ov }}$-values between 0.2 and 0.4 , with typical uncertainties of 0.1 to 0.3 , for five systems with OB-type components. Earlier on, Mermilliod \& Maeder (1986) made an extensive study of $\alpha_{\mathrm{ov}}$ needed to explain the HR diagrams of 25 young open clusters and they concluded that $\alpha_{\mathrm{ov}} \sim 0.3$ is needed to explain the observational data. This value was later revised to $\alpha_{\mathrm{ov}} \sim 0.2$ due to the use of other opacity computations (Meynet et al. 1993). These estimates from two independent classical methods are fully compatible with, but less precise than the seismic results. We note that they suffer from the same theoretical uncertainties as the seismology. 


\section{Future plans}

It is now well established seismically that core convective overshooting occurs in massive main sequence stars. The results summarised here were based on the fitting of only two to four $m=0$ oscillation modes. It is evident that a reduction in the uncertainty of the overshooting value will occur whenever several more well identified modes can be used to derive $\alpha_{\mathrm{ov}}$. This is one of the major goals for massive stars to be studied in the framework of the seismology programmes of the space missions CoRoT (Michel et al. 2006) launched on 27/12/2006 and Kepler (Christensen-Dalsgaard et al. 2007) to be launched in 2009 .

Large activities are continued to get more results from ground-based multi-site campaigns, in order to increase the sample with more evolved stars and stars of gradually faster rotation and higher mass-loss rate. The goal is to reach a sample that covers the evolution from the main sequence until the pre-supernova stage. In this way, stellar evolution models in the upper HR diagram can be calibrated seismically to a level of precision that has never been achieved before.

\section{References}

Aerts, C., Marchenko, S. V., Matthews, J. M., et al. 2006, ApJ, 642, 470

Aerts, C., Thoul, A., Daszyńska, J., et al. 2003, Science, 300, 1926

Aerts, C., Waelkens, C., Daszyńska-Daszkiewicz, J., et al. 2004, A\&A, 415, 241

Asplund, M., Grevesse, N., Sauval, A. J., et al. 2005, A\& A, 431, 693

Ausseloos, M., Scuflaire, R., Thoul, A., \& Aerts, C. 2004, MNRAS, 355, 352

Briquet, M. \& Aerts, C. 2003, A\&A, 398, 687

Briquet, M., Morel, T., Thoul, A., et al. 2007, MNRAS, 381, 1482

Christensen-Dalsgaard, J., Arentoft, T., Brown, T. M., et al. 2007, CoAst, 150, 350

Claret, A. 2007, A\&SA, 475, 1019

Cunha, M. S., Aerts, C., Christensen-Dalsgaard, J., et al. 2007, AESAR, 14, 217

De Ridder, J., Telting, J. H., Balona, L. A., et al. 2004, MNRAS, 351, 324

Dupret, M.-A., Thoul, A., Scuflaire, R., et al. 2004, A\&A, 415, 251

Grevesse, N. \& Noels, A. 1993, in: Prantzos, N., Vangioni-Flam, E., \& Casse, M. (eds.), Origin and evolution of the elements, (Cambridge: Cambridge University Press), 14

Handler, G., Shobbrook, R. R., \& Mokgwetsi, T. 2005, MNRAS, 362, 612

Kurtz, D. W. 2006, in Sterken, C., \& Aerts, C. (Eds.), Astrophysics of Variable Stars, (San Francisco: ASP) ASP Conf Ser, 349, 101

Mazumdar, A., Briquet, M., Desmet, M., \& Aerts, C. 2006, A\&A A, 459, 589

Mermilliod, J.-C. \& Maeder, A. 1986, A\& $A, 158,45$

Meynet, G., Mermilliod, J.-C., \& Maeder, A. 1993, A\&SAS, 98, 477

Michel, E., Baglin, A., Auvergne, M., et al. 2006, in Fridlund, M., Baglin, A., Lochard, J., \& Conroy, L. (Eds.), The CoRoT Mission, ESA-SP, 1306, 39

Miglio, A., Montalbán, J., \& Dupret, M.-A. 2007, MNRAS, 375, L21

Monteiro, M. J. P. F. G., Lebreton, Y., Montalban, J., et al. 2006, in Fridlund, M., Baglin, A., Lochard, J., \& Conroy, L. (Eds.), The CoRoT Mission, ESA-SP, 1306, 363

Moya, A., Christensen-Dalsgaard, J., Charpinet, S., et al. 2008, ApSS, in press (arXiv:0711.2587)

Pamyatnykh, A. A., Handler, G., \& Dziembowski, W. A. 2004, MNRAS, 350, 1022

Thoul, A., Scuflaire, R., Ausseloos, M., et al. 2004, CoAst, 144, 35

Zima, W. 2006, A\&A, 455, 227

\section{Discussion}

PRZYBILla: The pulsators often show chemical peculiarities. How would the differences relative to the solar mixture (which the asteroseismic models are based upon) influence the interpretation? 
AERTS: So far, we have too few frequencies to tune the individual abundances seismically. Rather, we derive the abundances from classical spectroscopy, determine the opacity, and check the mode excitation to see if we have consistency with the observed oscillations. If we have many well identified frequencies (several tens to hundreds), we will be able to tune the metal mixture seismically. We are still far from that in massive stars, but CoRoT and Kepler will hopefully help improve the situation in the coming decade

MAEDER: Can you tell us why you are also happy with the new solar abundances?

AERTS: 1) The seismic model of the Sun, even though it degraded somewhat in quality with the Asplund et al. (2005) abundances, is still very good! If I compare the error bars in the interior structure parameters of the Sun with those for massive stars, then I'd say there is not need for worries in this community. 2) As discussed in Miglio et al. (2007), some outstanding issues about mode excitation in B-type pulsators have now been solved, by using the OP opacities together with the new solar abundances. 3) For studies of massive stars, one may first as well use the B stars in the solar neighbourhood to compare with.

MEYNET: Do you have plans to do seismic studies of more rapid rotators?

AERTS: Yes, we have several multisite campaigns (finished and ongoing) focused on stars of higher $v \sin i$, but still only up to $v \sin i \simeq 60 \mathrm{~km} \mathrm{~s}^{-1}$. On the other hand, campaigns are also ongoing on young open clusters with B type pulsators (one such cluster is also in the FLAMES survey). Those have typically $v \sin i \sim 100-150 \mathrm{~km} \mathrm{~s}^{-1}$. Note, however, that the mode identification becomes very difficult for more rapid rotators, because the oscillation frequency multiplets are merged due to the rotational splitting, and they are therefore hard to disentangle.

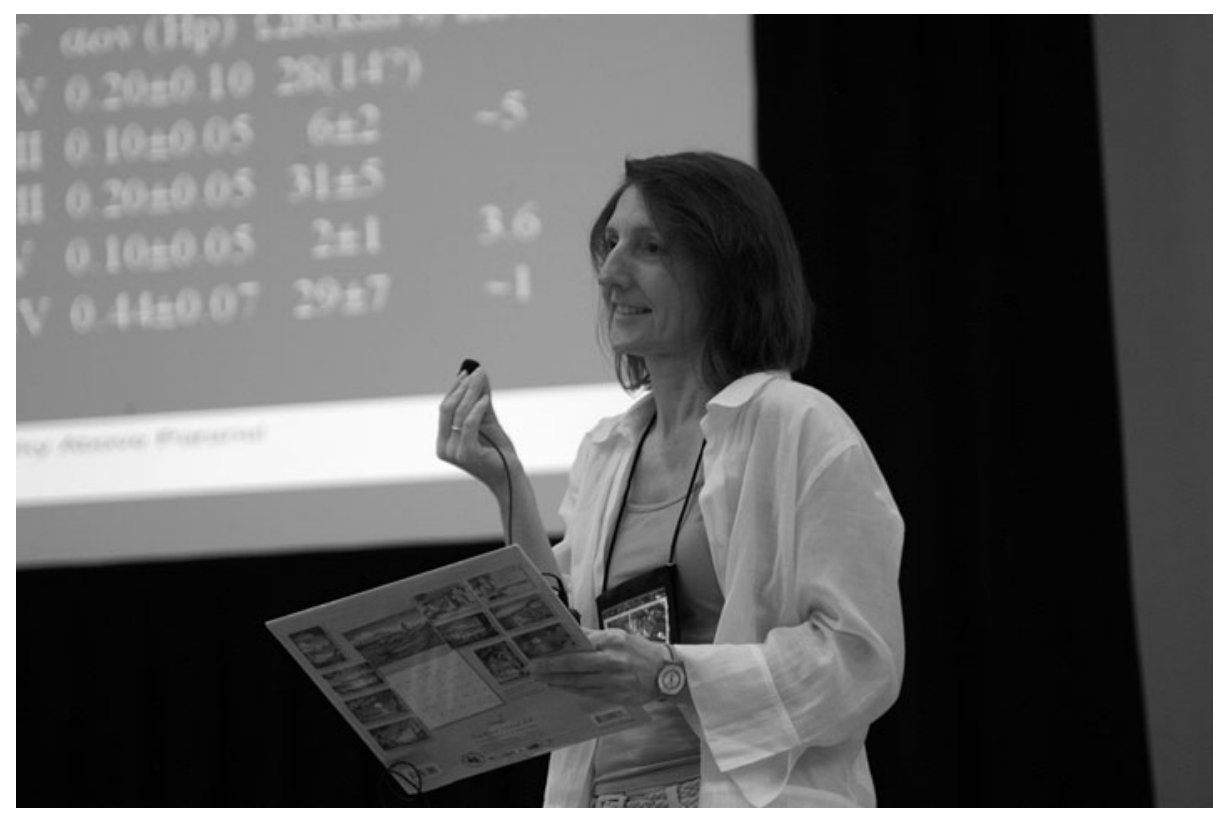

Conny Aerts. 


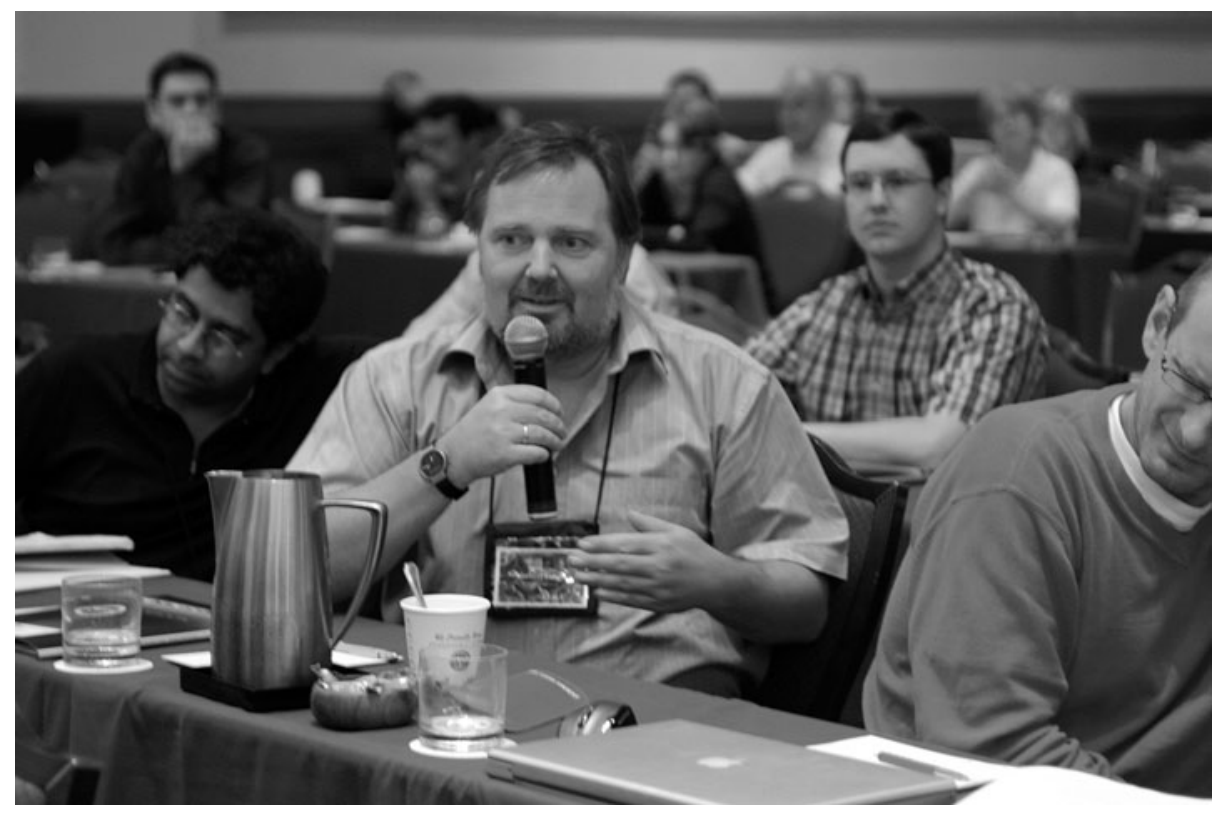

Norbert Langer.

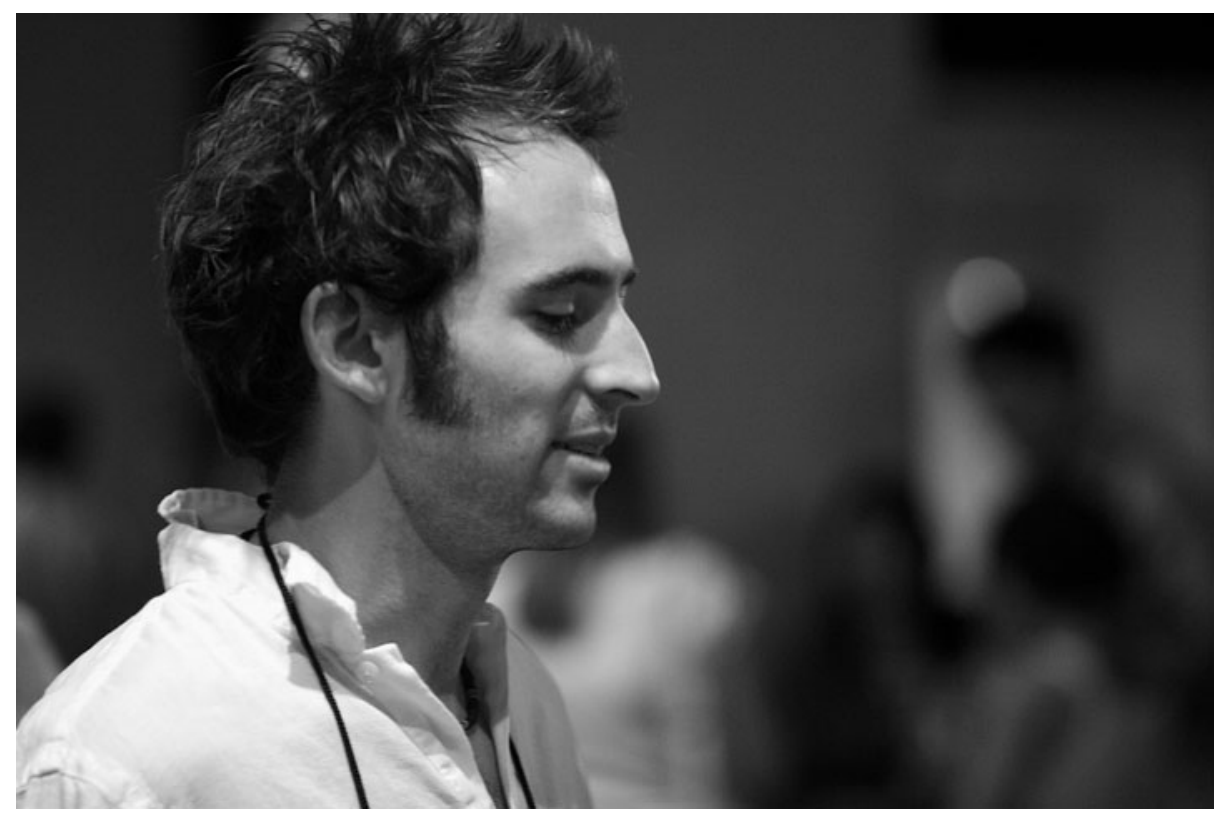

Ben Davies. 